\title{
To Sedate or Not to Sedate for Less Invasive Surfactant Administration: An Ethical Approach
}

\author{
Jennifer Peterson ${ }^{\mathrm{a}}$ Maria C. den Boer ${ }^{\mathrm{b}}$ Charles Christoph Roehr ${ }^{\mathrm{c}, \mathrm{d}, \mathrm{e}}$ \\ ${ }^{a}$ Neonatal Unit, St Mary's Maternity Hospital, Manchester Foundation Trust, Manchester, UK; ${ }^{b}$ Division of Neonatology,

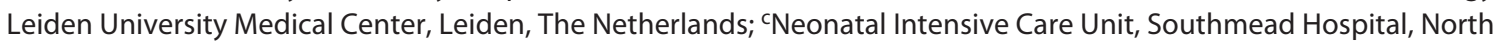 \\ Bristol Trust, Bristol, UK; ${ }^{~}$ Faculty of Health Sciences, University of Bristol, Bristol, UK; ${ }^{~}$ National Perinatal Epidemiology \\ Unit, Clinical Trials Unit, Nuffield Department of Population Health, Medical Sciences Division, University of Oxford, \\ Oxford, UK
}

\section{Keywords}

Neonates · Respiratory depression · Surfactant · Ethics

\begin{abstract}
Less invasive surfactant administration (LISA) is an effective, minimally invasive technique of administering surfactant to infants with respiratory distress syndrome. While termed less invasive, LISA still requires airway instrumentation with direct laryngoscopy, thus may be considered painful. However, the issue of whether or not to routinely sedate infants for LISA remains contentious, with significant variation in practice between centres. Proponents for giving pharmacological analgesia and/or sedation predominantly focus on patient comfort during the procedure. However, those who favour non-pharmacological measures of pain management focus on the potential for procedural success without the risk of adverse events, such as respiratory depression and potentially the need for escalation to intubation, which may occur with pharmacological agents. The neonatal population who may benefit from LISA is varied. Due to this variety in presentation type, gestational age, and unit experience, there is a need to provide an individualized, tailored approach to sedation and analgesia for these infants. Using a
\end{abstract} posed to sedative medications on the assumption of potential distress, rather than in response to signs of actual distress. This places the infant at risk of the adverse reactions, potentially without them ever having needed the beneficial effect of the medications. This seems an unnecessary risk. This article explores the ethical arguments pertaining to analgesia and sedation during the LISA technique, concluding that a standardized approach to the usage of pharmacological sedation is undesirable. Moreover, we maintain that procedural analgesia and sedation should be based on individualized, infant-centred assessment, rather than on a rigid, standardized approach.

(c) 2021 S. Karger AG, Basel

\section{Introduction}

Preterm infants, with their inherently fragile lungs, stand to accrue ventilator-associated lung disease [1]. Bronchopulmonary dysplasia is a debilitating disease of preterm infants especially prevalent in those ventilated for prolonged periods [2]. Therefore, mechanical ventilation should be avoided unless absolutely necessary [3].

Correspondence to:

Charles Christoph Roehr, charles.roehr@bristol.ac.uk 
Fig. 1. Considerations when performing LISA. LISA, less invasive surfactant administration; NICU, neonatal intensive care unit.

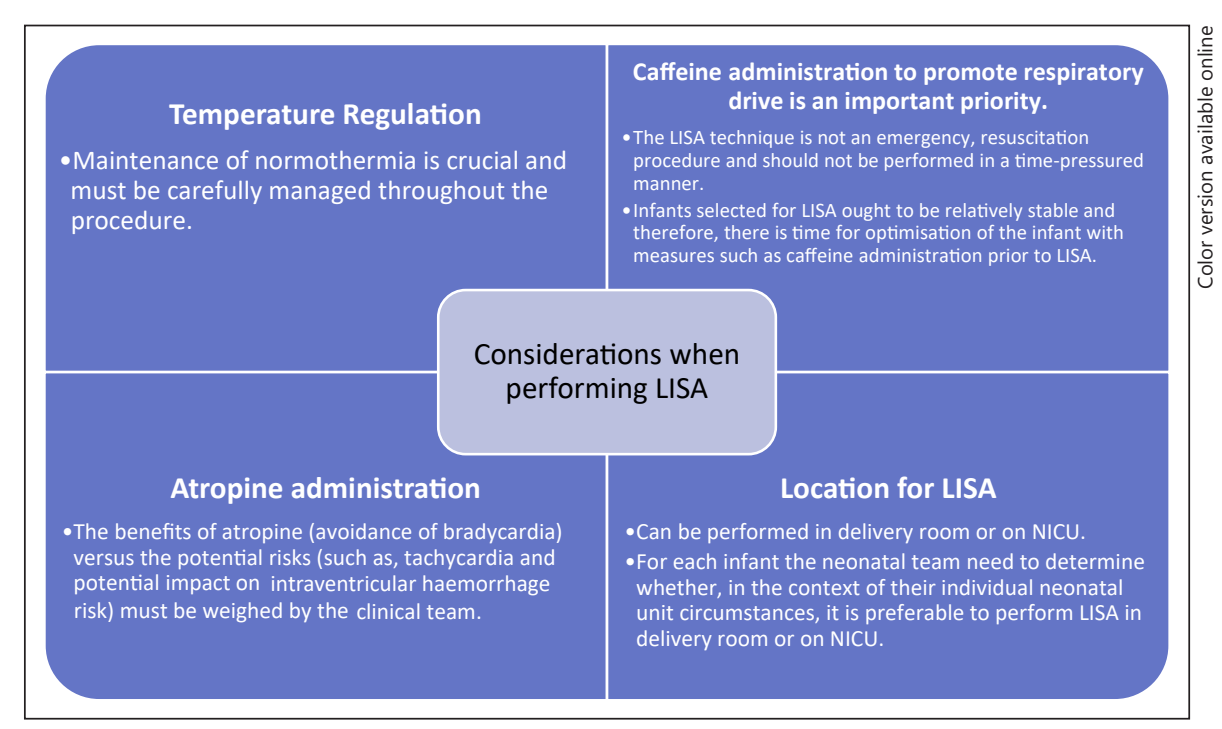

Conversely, it is equally recognized that many preterm infants require postnatal exogenous surfactant replacement [4]. Traditionally, surfactant is administered through an endotracheal tube (ETT), placed beneath the vocal cords by the technique of direct laryngoscopy (endotracheal intubation). However, subjecting infants with good respiratory drive, stabilized on non-invasive respiratory support, to the process of intubation in order to administer surfactant seems unfortunate. Instead, a variety of techniques have been developed to provide surfactant while avoiding endotracheal intubation. The less invasive surfactant administration (LISA) technique is a minimally invasive method to provide surfactant to infants with respiratory distress syndrome without the use of an ETT $[5,6]$. Current evidence suggests LISA is effective in reducing the need for endotracheal intubation, mechanical ventilation, bronchopulmonary dysplasia, and death [7-10].

Despite convincing evidence, there is significant variation in adoption of the LISA technique in neonatal intensive care units [11-14]. Performing the LISA technique successfully and safely requires robust multidisciplinary coordination of care. There are numerous elements of the LISA technique that require careful clinical consideration (Fig. 1). The fear of potential patient harm can drive a heightened sense of apprehension when a new technique involves the preterm infant airway. Therefore, significant variation in the use of pharmacological agents to provide infants with analgesia and sedation during the LISA procedure was found among neonatal centres world-wide [13]. While some centres have opted for a standard approach using medications for every infant undergoing
LISA, others try to avoid using pharmacological sedation entirely. Furthermore, for centres using sedative and analgesic medications for LISA, there remains a wide variety of choices for the specific agent used, from fentanyl and morphine, through to propofol and ketamine $[13,15]$.

This article explores the ethical approach to analgesia and sedation during the LISA technique. The LISA technique, as with all treatment options, has indications and limitations. Selection of unsuitable infants may increase the risk of LISA failure and contribute to poorer overall outcome $[16,17]$. The discussion around the ethical aspects of sedation (or not) for LISA in this article relates to infants that have been appropriately selected for the LISA technique. We argue that a standardized approach to the usage of pharmacological sedation is undesirable. Procedural analgesia and sedation for infants should be based on individualized assessment, rather than a rigid, standardized approach.

\section{First Principle: Do No Harm}

The LISA technique poses a conundrum to clinicians in regard to the edict of primum non nocere, "First, Do No Harm" [18]. The current verified techniques for surfactant administration, either by intubation or the LISA technique, both require manipulation of the airway with a laryngoscope. Insertion of the laryngoscope blade into the airway is known to cause systemic hypertension, bradycardia, hypoxaemia, and raised intracranial pressure through stimulation of the vagal nerve [19]. Therefore, most neonatal units would advocate for premedication to 
sedate the infant while intubating. This provision of analgesia and sedation aids the comfort of the infant and improves the likelihood of procedure success. In the case of intubation, the infant receives surfactant via the ETT. As a routine part of the intubation procedure, the infant would be placed on a ventilator for a variable period of time. Therefore, if the pre-medications sedate the infant to the extent they lose their respiratory drive, this is not problematic as the now apnoeic infant is connected to the ventilator.

In the case of LISA, a crucial benefit of the procedure lies in avoiding apnoea and mechanical ventilation. The technique relies on the infant maintaining their own respiratory drive. However, the clinical team may be concerned that to attempt LISA without sedative/analgesic medication would cause excessive distress to the infant. The infant may then resist the procedure, causing additional distress which may lead to procedure failure and consequently, intubation. Therefore, the clinical team may opt to administer sedative/analgesic medication. However, if pharmacological sedation is administered and the infant loses its respiratory drive, the infant will require further manipulation of the airway as the team, after stabilizing the infant with intermittent positive pressure ventilation (IPPV), may well switch to emergency intubation to administer the required surfactant. The infant then loses the benefit of avoiding mechanical ventilation and has been put through significant airway instrumentation on at least 2 occasions. Alternatively, the team may consider administration of naloxone to reverse the effects of the sedation and may then reassess the infant for suitability for repeat attempt at LISA or intubation. In this situation the infant has experienced significant decompensation and additionally, there is limited research into the short and long-term side effects of naloxone, particularly in premature infants $[20,21]$. Therefore, the clinical conundrum lies in balancing the harm intrinsic to the LISA technique with the risk of harm implicit in provision of sedation for the procedure, with the most undesirable risk here being respiratory depression secondary to pharmacological sedation. In this situation, the treating clinician may feel there is harm with either option. The respective weight of each of these harms will now be considered.

\section{Weighing the Potential Harms}

\section{Pain and Distress}

Until relatively recently, premature neonates were considered unlikely to perceive pain due to an underde- veloped nervous system. This contention is being reversed, with an ever-increasing body of scientific evidence showing that neonates are capable of feeling pain [22]. Infants at birth acquire human rights, including the right to not be subject to harm. While for older children and adults there may be a meaningful emotional distinction between pain and distress, this is less distinct for infants. Both pain and distress are unpleasant, noxious experiences. Painful and distressing stimuli are known to have a negative effect on the developing nervous system $[23,24]$. For a neonate, the sensation of either pain or non-painful distress can lead to increased cortisol, tachycardia, desaturation, and potentially significant deterioration. Given that either pain or distress may lead to deterioration of the infant, then avoidance of both should be a priority when providing care. However, where, for the overall health of the infant, pain or distress cannot be avoided, then measures should be taken to minimize the impact of these unpleasant stimuli on the infant.

\section{Role of Pharmacological and Non-Pharmacological Options}

Non-pharmacological and pharmacological agents can be used to minimize the impact to the individual of a necessary but noxious stimulus. In the case of non-pharmacological methods, such as containment holding, swaddling, and sucrose, the clinician must ensure these adequately cover the infant's needs. In the case of pharmacological agents, such as opioids, the clinician must ensure the drug and dose meet the infant's requirement without excessive administration, which could lead to unintended negative effects. Analgesic and sedative measures must always be in proportion to the level of distress experienced by the infant. For example, for an infant undergoing surgery, there would be an expectation that this would confer significant pain and, therefore, non-pharmacological measures would not be sufficient and pharmacological measures should be employed. However, while there may be an expected level of pharmacological analgesia required depending on the extent of the operation, the administration of analgesia should be tailored to the infant's response. The aim is to offer the infant an adequate level of analgesia to ensure that they are comfortable, without providing excessive exposure to the analgesic drug that they develop undesirable effects [25]. In the case of opioids, this may be entirely appropriate following invasive surgery. However, if continued at high doses or for a prolonged period of time following the operation, then the infant may develop unwanted effects such as withdrawal when the opioid is eventually stopped [26]. 
Additionally, when pharmacological agents are required in order to alleviate infant suffering, this does not mean that there is not a concurrent role for non-pharmacological interventions. Ensuring an infant is well positioned, providing containment holding and, where appropriate, provision of sucrose may allow for pharmacological agents to be provided at lower doses or for shorter periods. As such, the infant is exposed to a reduced risk of undesirable side effects, both short- and long-term, or complications from pharmacological agents. In the case of LISA, in order to justify providing analgesia or sedation, it must be established whether the technique causes pain or distress.

\section{Perceptions of Airway Instrumentation}

Instrumentation of the airway has associated negative effects. However, in the cases of apnoea or moderate-severe respiratory distress syndrome, airway instrumentation may be necessary to ensure avoidance of a greater harm to the patient; deterioration and death. Perceptions of airway instrumentation are often viewed through an adult lens. However, when considering a procedure through this lens, it becomes difficult to remove the impact of adult cognisance from this perspective. Adults have the ability to form meaningful, accessible memories. These memories are crafted from the context and emotions surrounding the event. They are rarely an entirely factual, non-emotional account of events. In studies focussing on adult patients who experienced rapid sequence induction (RSI) and intubation (an acute, emotionally fraught, high-risk situation), the adult recollection of intubation was that intubation was painful and deeply unpleasant [27]. Interestingly, in studies of adult intubation in different contexts, the perception of the intubation itself was much more tolerable. Adult patients undergoing "awake intubation" due to having a high-risk airway described their experiences differently to those undergoing RSI intubation [28]. One would imagine that having the label of high-risk airway and undergoing awake intubation would be stressful and disturbing as an experience. However, the interviewed patients described that once they understood the reasons behind the need for them to be awake and the intubation procedure itself, the reality of the awake intubation experience was tolerable and not painful [28].

Clearly, for the neonatal population, there is little benefit to having a reassuring discussion of the intubation process with the neonate themselves. However, these adult perceptions of intubation have relevance to neonates for two reasons. The first is that these studies suggest that intubation itself is not intrinsically painful, but can be highly distressing, particularly when performed in an unexpected emergency situation. This highlights the second point of relevance: adult perception of intubation. While intubation will always be unexpected for neonatal patients, their inability to attach contextual significance to the situation means they may not be as distressed as an adult may imagine if the adult were to picture themselves in the same situation. The clinicians treating the neonate are likely to consider intubation from an adult perspective and may conclude that they would find the procedure highly painful and distressing and therefore, so would a neonatal patient. Neonatal patients have no ability to understand that a particular procedure is about to occur and have no awareness of the severity of their own condition. Therefore, they will not feel any anticipatory distress; even though their treating clinical team may feel this on their behalf. Granted, the neonate may experience distress during the airway instrumentation. For the LISA technique, while there is a similar level of instrumentation of the airway to intubation, the inserted catheter is considerably smaller, filling much less of the airway. While there is no true adult equivalent to the LISA catheter and therefore, no adult descriptive data to compare the experience, one would imagine having a LISA catheter in place would be preferable to an ETT. There are trials in development to test this theory in the neonatal population [29]. Currently though, there is reasonable justification for expecting that airway instrumentation of any sort could elicit a spectrum of responses, from being simply unpleasant to potentially extremely distressing for a neonate. Certainly, the experience described by patients undergoing flexible bronchoscopy, which involves airway instrumentation and is therefore likely to be the closest adult comparator to LISA, is reported as unpleasant and painful by many adults who have undergone this procedure [30]. Given this risk of potential pain and distress to the infant secondary to airway instrumentation, the treating clinician's focus should be to ensure that the neonate is calm and not displaying signs of pain or distress at any point during the procedure. By making serial pain assessments throughout LISA, the team can start with a level of analgesia matched to the infant (e.g., non-pharmacological measures) and then escalate their management should the infant display any unanticipated signs of pain or distress. Interventions should be matched to the individual level of distress exhibited by the infant. 


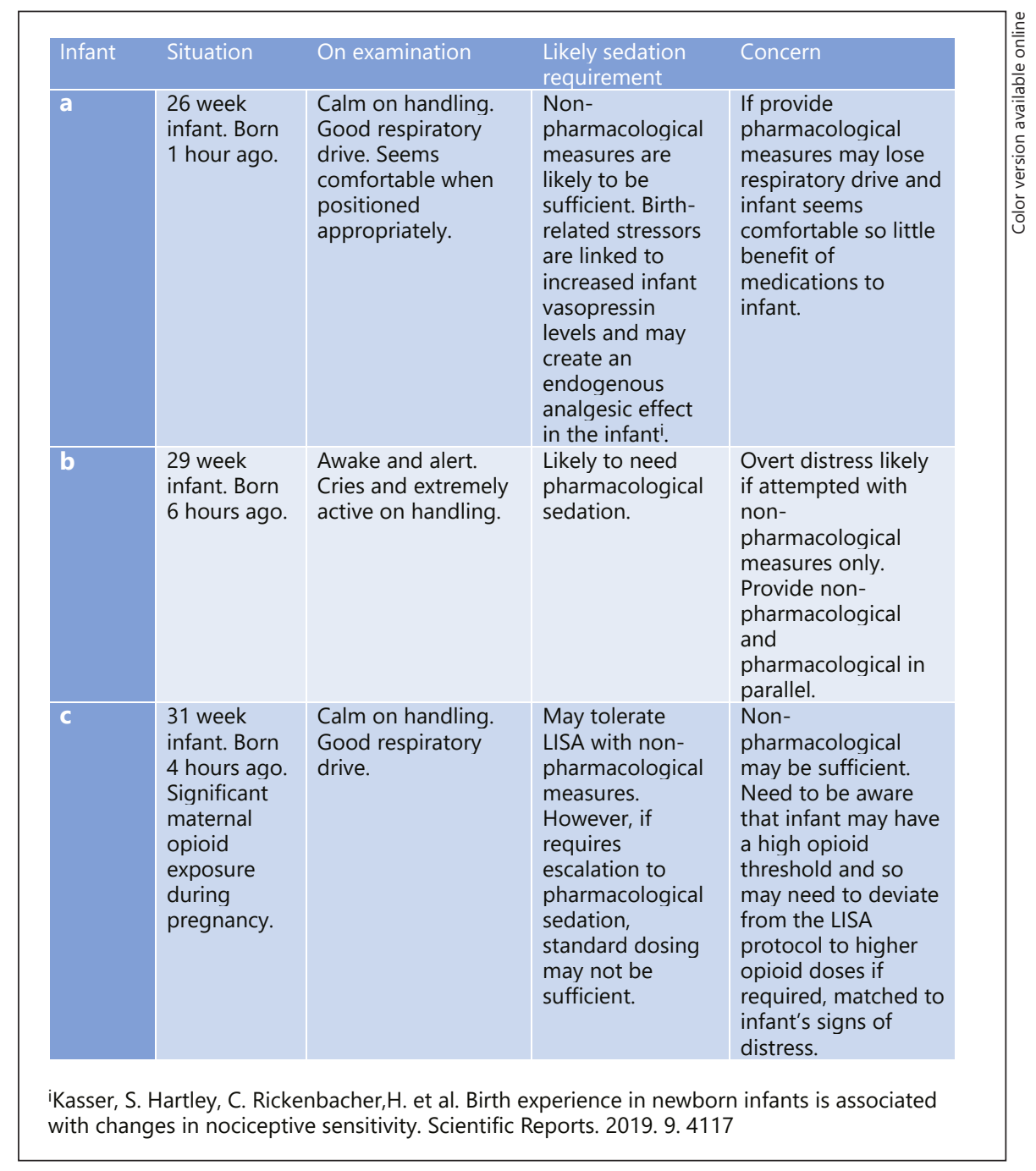

Fig. 2. Clinical vignettes [32].

\section{The Problem of Standardized Approaches}

Providing pharmacological agents to infants undoubtedly carries risk [31]. Providing the procedure is being performed in the infant's best interests, then if the infant demonstrates signs of distress when preparing for, or during, the procedure, it becomes justifiable to administer the medication to alleviate the suffering. However, the provision of a standardized dose of pharmacological analgesia or sedation does not ensure the infant will not experience any distress. LISA protocols that contain sedation medications will often have a standardized dose. Applied to individual infants, there is no assurance that the recommended dose will be adequate for that infant. For example, a unit may have a standard regime for fentanyl use for LISA. This may be adequate or even excessive for some infants. However, there may be other infants, for example, infants exposed to opioids during pregnancy, where the routine dosing of fentanyl is entirely inadequate. If there is a rigid standardized protocol then the treating team may be reluctant to deviate from this, despite the fact that the provided analgesia is not meeting that infant's requirements.

\section{An Individualized Approach}

Individual infants, like older children and adults, will have different personalities and different levels of reaction to the same stimulus. Recognizing infants as individuals requires staff to manage their needs with an individualized approach, rather than a rigid standardized response. 
The clinical vignettes shown in Figure 2 are taken from the first author's clinical experiences with LISA and aim to exemplify that the range of infants who serve to benefit from LISA are diverse. Therefore, there needs to be a diverse range of approaches used in ensuring their individual comfort during the procedure, rather than a blanket approach. Clinicians should be encouraged to assess signs of distress and tailor management to this, rather than exposing every infant undergoing LISA within the unit to an inflexible, uniform sedative regime, which may not have been needed.

This individualized approach is reliant on individual clinician assessment of the infant's distress. This carries the potential risk that some clinicians may inaccurately appreciate the extent of the infant's distress [3235]. This risk may be more present if the clinician is less experienced in infant pain assessment, if the clinician is apprehensive about performing the LISA procedure itself or if there is excessive clinician concern regarding the risk of respiratory depression from pharmacological agents [36]. While this risk is present, LISA should always be undertaken with senior clinical support, both medical and nursing [6]. Serial assessments of the infant's level of distress pre and during LISA should form a key part of the procedure process and unit protocol [37]. Incorporation of neonatal pain assessment tools, for example, COMFORTneo $[38,39]$ would be prudent to ensure a full assessment of the infant and attempt to standardize the assessment process across different infants and different staff teams providing LISA [39-41]. Neonatal pain assessment tools are useful but should be correlated with senior medical and nursing experience. This is important as some elements of pain scoring systems may be non-specific, for example, an increased heart rate may be secondary to pain or to infection [42]. Additionally, elements of the pain score may be difficult to assess, such as brow furrowing during instrumentation of the airway, and therefore, clinicians may rely on other distress parameters, such as the infant's movement and activity level during the procedure. By utilizing an objective pain scoring tool alongside a multidisciplinary team assessment of the infant's distress (if any), this approach should reduce the potential for clinician bias or misinterpretation to impact the infant and their potential pain. This multidisciplinary assessment throughout the LISA procedure should ensure the provision of analgesia and sedation is matched appropriately and individually to the infant. This individualized approach is applicable wherever the LISA procedure is being at- tempted as the same ethical arguments apply regardless of whether LISA is being performed in delivery suite or within the neonatal unit. As stated earlier in this article, LISA is not an emergency procedure. Therefore, there should be adequate time for optimization of the infant's care, including assessment of the infant's handling and pain scores prior to and during the LISA procedure.

\section{Learning to LISA}

The discussion so far has focused on the individuality of the infant and matching the level of sedation to that specific infant. One complication to this argument is the expertise of the treating physician who will have their own individual level of experience in performing the LISA technique.

The LISA procedure is generally not suitable for junior clinicians inexperienced in obtaining a clear view of the vocal cords. The justification here is that instrumentation of the airway during LISA should be short lived in order to avoid deterioration and preserve the infant's own respiratory effort as much as possible. Consequently, LISA should be performed by senior clinicians who are more likely able to promptly locate an adequate view of the cords, insert the LISA catheter and then remove the laryngoscope blade without dislodging the newly inserted catheter. However, even where the treating clinician is experienced, there will remain a period of time where the LISA technique will be a new procedure and therefore, unfamiliar to them. While simulation training can assist in aiding clinicians to develop hands-on experience with the technique, it does not entirely replicate the experience of performing the technique on a real infant. There may be increased anxiety, regardless of clinician seniority, when implementing a new technique, especially when high risk, which procedures involving the infant airway inherently are. In these circumstances, the balance is not simply between the risk of infant distress during LISA versus the risk of infant deterioration due to respiratory depression following sedation, but also, the potential risk of increased procedure length and clinician error given that the technique is new.

In these cases, the clinician performing LISA may feel uneasy about attempting to perform the new technique, coordinate the team and simultaneously ensure they have adequately and accurately assessed the infant's level of distress. Clinicians may feel that, on balance, sedation should be used regardless of the infant's level of distress 
prior to starting the procedure. However, as stated previously, infants should not be exposed to the side effects of medications where there is not a clear indication for administering them. Clinician apprehension is not sufficient justification.

The LISA technique must be viewed as a multidisciplinary procedure. It requires close assessment and monitoring of multiple infant parameters (respiratory drive, vital signs, temperature control, and pain scores) and therefore cannot be safely performed by only one clinician. Particularly, in circumstances where the LISA technique is new to the clinician and/or neonatal unit, it is important that the technique be implemented in a calm, coordinated manner. The technique requires a senior clinician with experience in airway management to perform the airway instrumentation and LISA catheter insertion. Therefore, this individual clinician should not also take on the role of coordinating the team and assessing the pain scores. This clinician may well contribute to this assessment if they feel able. However, there must be additional team members who are assigned these roles, and who are charged with communicating their assessments to a designated team leader for the LISA procedure. By following this structure, the clinician performing the airway elements of the LISA technique is able to focus on this and does not have to also carry the mental task of singlehandedly assessing for pain and coordinating the team. This shared responsibility approach ensures safety for the infant, while allowing an individualized approach to the infant's sedation and therefore does not needlessly expose the infant to risk of side effects from sedative medications simply to alleviate the clinician apprehension around performing the new technique.

\section{Final Thoughts}

Each unit should develop expertise with various nonpharmacological and pharmacological options in order to ensure the infant remains comfortable throughout the procedure. The initial position should be for non-pharmacological methods to ensure infant comfort. Escalation to pharmacological agents should only occur if nonpharmacological methods have been insufficient to ensure infant comfort, as shown by signs of distress from the infant assessed by the multidisciplinary team. This escalation-based approach ensures infant comfort, minimizes staff apprehension, and increases the likelihood of the LISA technique being successful, without exposing every infant to potentially harmful and often unnecessary medications. One set approach to sedation does not fit all.

\section{Conflict of Interest Statement}

There are no conflicts of interest to declare from any of the authors.

\section{Funding Sources}

No funding received.

\section{Author Contributions}

Peterson devised and wrote the initial article. Boer reviewed and contributed to the revised article. Roehr devised and supervised the project. All authors approved the final manuscript as submitted and agree to be accountable for all aspects of the work.

\section{References}

1 Donn SM, Sinha SK. Minimising ventilator induced lung injury in preterm infants. Arch Dis Child Fetal Neonatal Ed. 2006;91:F22630.

2 Brew N, Hooper S, Zahra V, Wallace M, Harding R. Mechanical ventilation injury and repair in extremely and very pretem lungs. PLoS One. 2013;8:e63905.

3 Ho JJ, Subramaniam P, Davis PG. Continuous positive airway pressure (CPAP) for respiratory distress in preterm infants. Cochrane Database Syst Rev. 2020 Oct 15;10(10) CD002271.

4 Banerjee S, Fernandez R, Fox GF, Goss KCW, Mactier H, Reynolds P, et al. Surfactant replacement therapy for respiratory distress syndrome in preterm infants: United King- dom National Consensus. Pediatr Res. 2019; 86:12-4.

5 Dargaville PA, Aiyappan A, De Paoli AG, Kuschel CA, Kamlin CO, Carlin JB, et al. Minimally-invasive surfactant therapy in preterm infants on continuous positive airway pressure. Arch Dis Child Fetal Neonatal Ed. 2013; 98:F122-6.

6 Vento M, Bohlin K, Herting E, Roehr CC Dargaville PA. Surfactant administration via thin catheter: a practical guide. Neonatology. 2019;116:211-26.

7 Sweet DG, Carnielli V, Greisen G, Hallman M, Ozek E, te Pas A, et al. European consensus guidelines on the management of respiratory distress syndrome: 2019 update. Neonatology. 2019;115:432-50.
8 National institute for Health and Care Excellence (NICE). Specialist neonatal respiratory care for babies born preterm. NICE guidelines 124. London: National Institute for Health and Care Excellence; 2019.

9 Klebermass-Schrehof K, Wald M, Schwindt J, Grill A, Prusa AR, Haiden N, et al. Less invasive surfactant administration in extremely preterm infants: impact on mortality and morbidity. Neonatology. 2013;103(4):252-8.

10 Aldana-Aguirre JC, Pinto M, Featherstone RM, Kumar M. Less invasive surfactant administration versus intubation for surfactant delivery in preterm infants with respiratory distress syndrome: a systematic review and meta-analysis. Arch Dis Child Fetal Neonatal Ed. 2017;102:F17-23.
An Ethical Approach to Sedation for Less Invasive Surfactant Administration
Neonatology 2021;118:639-646 DOI: $10.1159 / 000519283$ 
11 Beltempo M, Isayama T, Vento M, Lui K, Kusuda S, Lehtonen L, et al. Respiratory management of extremely preterm infants: an international survey. Neonatology. 2018;114:2836.

12 Klotz D, Porcaro U, Fleck T, Fuchs H. European perspective on less invasive surfactant administration-a survey. Eur J Pediatr. 2017; 176:147-54.

13 Bhayat S, Kaur A, Premadeva I, Reynolds P, Gowda H. Survey of less invasive surfactant administration in England, slow adoption and variable practice. Acta Paediatr. 2020; 109:505-10.

14 Kurepa D, Perveen S, Lipener Y, Kakkilaya V. The use of less invasive surfactant administration (LISA) in the United States with review of the literature. J Perinatol. 2019;39:426.

15 Herting E, Härtel C, Göpel W. Less invasive surfactant administration: best practices and unanswered questions. Curr Opin Pediatr. 2020;32:228-34.

16 Sokol D. "First do no harm": revisited. BMJ. 2013;347:F6426.

17 Ayed M, Shah VS, Taddio A. Premedication for non-urgent endotracheal intubation for preventing pain in neonates. Cochrane Database Syst Rev. 2017;2:CD012562.

18 Marchant A. "Neonates do not feel pain": a critical review of the evidence. Biosci Horiz Int J Stud Res. 2014;7:hzu006.

19 Valeri BO, Holsti L, Linhares MBM. Neonatal pain and developmental outcomes in children born preterm. Clin J Pain. 2015;31(4):355-62.

20 Williams MD, Lascelles BDX. Early neonatal pain-a review of clinical and experimental implications on painful conditions later in life. Front Pediatr. 2020;8:30.

21 Schiller RM, Allegaert K, Hunfeld M, van den Bosch GE, van den Anker J, Tibboel D. Analgesics and sedatives in critically ill newborns and infants: the impact on long-term neurodevelopment. J Clin Pharmacol. 2018;58(Suppl 10):S140-50.
22 Kimball D, Kincaide RC, Ives C, Henderson $\mathrm{S}$. Rapid sequence intubation from the patient's perspective. West J Emerg Med. 2010; 12:365-7.

23 Knudsen K, Nilsson U, Högman M, Pöder U. Awake intubation creates feelings of being in a vulnerable situation but cared for in safe hands: a qualitative study. BMC Anesthesiol. 2016;16:71.

24 US National Library of Medicine. Stress assessment with and without analgesia during surfactant therapy in preterm infants. ClinicalTrials.gov. 2021 Apr 12. https://clinicaltrials.gov/ct2/show/NCT04073173.

25 Schiller RM, Allegaert K, Hunfeld M, van den Bosch GE, van den Anker J, Tibboel D. Analgesics and sedatives in critically ill newborns and infants: the impact on long-term neurodevelopment. J Clin Pharmacol. 2018;58 (Suppl 10):S140-50.

$26 \mathrm{McPh}$ rson C. Sedation and analgesia in mechanically ventilated preterm neonates: continue standard of care or experiment? J Paediatr Phamacol Ther. 2012;17(4):351-64.

27 Breau LM, McGrath PJ, Stevens B, Beyene J, Camfield CS, Finley GA, et al. Healthcare professionals' perceptions of pain in infants at risk for neurological impairment. BMC Pediatr. 2004;4:23.

28 Suciu LM, Cucerea M, Simon M, Avasiloaiei A, Petrescu O, Andrei SB. Health care professional's attitude towards the effective management of pain in the critically ill neonate. J Crit Care Med. 2015;1(3):107-12.

29 Lago P, Garetti E, Merazzi D, Pieragostini L, Ancora G, Pirelli A, et al. Guidelines for procedural pain in the newborn. Acta Paediatr. 2009;98:932-9.

30 Emanuel D. Comfort-scale and newborns: a review. Paediatr Res. 2010;68:125.

31 Maaskant J, Raymakers-Janssen P, Veldhoen E, Ista E, Lucas C, Vermeulen H. The clinimetric properties of the COMFORT scale: a systematic review. Eur J Pain. 2016;20:1587611.
32 Kasser S, Hartley C, Rickenbacher H, Klarer N, Depoorter A, Datta AN, et al. Birth experience in newborn infants is associated with changes in nociceptive sensitivity. Sci Rep. 2019 Mar;9(1):4117.

33 Desai A, Aucott S, Frank K, Silbert-Flagg J. Comparing N-PASS and NIPS: improving pain measurement in the neonate. Lippincott Nursing Center. Available from: https:// www.nursingcenter.com/ce_articleprint? an $=00149525-201808000-00005$.

34 Mathews L. Pain in children: neglected, unaddressed and mismanaged. Indian J Palliat Care. 2011;17(Suppl 1):S70-3.

35 Perry M, Tan Z, Chen J, Weidig T, Xu W, Cong XS. Neonatal pain: perceptions and current practice. Crit Care Nurs Clin North Am. 2018;30:549-61.

36 Witt N, Coynor S, Edwards C, Bradshaw H. A guide to pain assessment and management in the neonate. Curr Emerg Hosp Med Rep. 2016;4:1-10.

37 Perry M, Tan Z, Chen J, Weidig T, Xu W, Cong XS. Neonatal pain: perceptions and current practice. Crit Care Nurs Clin North Am. 2018;30:549-61.

38 Perry M, Tan Z, Chen J, Weidig T, Xu W, Cong XS. Neonatal pain: perceptions and current practice. Crit Care Nurs Clin North Am. 2018;30:549-61.

39 Victoria N, Murphy A. Exposure to early life pain: long term consequences and mechanisms. Curr Opin Behav Sci. 2016;7:61-8.

40 BAPM Working Group. Perinatal management of extreme preterm birth before 27 weeks of gestation: a framework for practice. Br Assoc Perinatal Med. 2019.

41 Mathew PJ, Mathew JL. Assessment and management of pain in infants. Postgrad Med J. 2003;79:438-43.

42 Sullivan M. Ethical principles in pain management. Pain Med. 2000;1(3):274-9. 